\title{
Using ZINC08918027 inhibitor to determine Aurora kinase-chromosomal passenger complex isoforms in mouse oocytes
}

\author{
Caroline Kratka ${ }^{1}$, David Drutovic ${ }^{2}$, Cecilia S. Blengini ${ }^{1,3}$ and Karen Schindler ${ }^{1,3^{*}}$ (D)
}

\begin{abstract}
Objective: Miscarriages affect $10 \%$ of women aged $25-29$, and $53 \%$ of women over 45 . The primary cause of miscarriage is aneuploidy that originated in eggs. The Aurora kinase family has three members that regulate chromosome segregation. Therefore, distinguishing the roles of these isoforms is important to understand aneuploidy etiology. In meiosis, Aurora kinase A (AURKA) localizes to spindle poles, where it binds TPX2. Aurora kinase C (AURKC) localizes on chromosomes, where it replaces AURKB as the primary AURK in the chromosomal passenger complex (CPC) via INCENP binding. Although AURKA compensates for CPC function in oocytes lacking AURKB/C, it is unknown whether AURKA binds INCENP in wild type mouse oocytes. ZINC08918027 (ZC) is an inhibitor that prevents the interaction between AURKB and INCENP in mitotic cells. We hypothesized that ZC would block CPC function of any AURK isoform.

Results: ZC treatment caused defects in meiotic progression and spindle building. By Western blotting and immunofluorescence, we observed that activated AURKA and AURKC levels in ZC-treated oocytes decreased compared to controls. These results suggest there is a population of AURKA-CPC in mouse oocytes. These data together suggest that INCENP-dependent AURKA and AURKC activities are needed for spindle bipolarity and meiotic progression.
\end{abstract}

Keywords: Aurora kinase, Oocyte, Meiosis, Chromosomal passenger complex

\section{Introduction}

The conserved serine/threonine protein kinases in the Aurora kinase (AURK) family play critical roles in regulating chromosome segregation in many cell types $[1,2]$. Unlike non-mammalian eukaryotes which encode one or two Aurk homologs, the mammalian genome encodes three [3, 4]. AURKA and AURKB isoforms are expressed in both mitotic and meiotic cells, whereas the AURKC isoform is expressed mainly in meiotic cells. In mouse oocytes, AURKA localizes to spindle poles and microtubules by binding its activator, TPX2 $[5,6]$ where it is important for normal spindle formation [7]. AURKC is

\footnotetext{
*Correspondence: ks804@hginj.rutgers.edu

${ }^{1}$ Department of Genetics, Rutgers University, The State University of New Jersey, 145 Bevier Rd, Piscataway, NJ 08854, USA

Full list of author information is available at the end of the article
}

the catalytic subunit of the meiotic Chromosomal Passenger Complex (CPC), where it binds the scaffolding unit INCENP (Inner Centromere Protein) and localizes to chromatin. AURKC is responsible for most functions that AURKB-CPC has in mitosis $[8,9]$, including regulating chromosome alignment and correcting erroneous kinetochore-microtubule attachments [10-12]. As a result, AURKB is diffuse in the oocyte cytoplasm and has still to be defined mechanisms in protecting egg quality with age $[13,14]$. Therefore, subcellular localization and function of AURKA and AURKC in mouse oocytes is dictated by their binding to different activators as in mitotic cells.

Analyses of oocyte-specific mouse knockout strains reveals complex genetic interactions amongst the kinases that appear to be unique to the female germline. For example, AURKC negatively regulates AURKA by

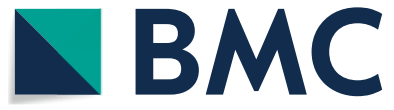

(c) The Author(s) 2022. Open Access This article is licensed under a Creative Commons Attribution 4.0 International License, which permits use, sharing, adaptation, distribution and reproduction in any medium or format, as long as you give appropriate credit to the original author(s) and the source, provide a link to the Creative Commons licence, and indicate if changes were made. The images or other third party material in this article are included in the article's Creative Commons licence, unless indicated otherwise in a credit line to the material. If material is not included in the article's Creative Commons licence and your intended use is not permitted by statutory regulation or exceeds the permitted use, you will need to obtain permission directly from the copyright holder. To view a copy of this licence, visit http://creativecommons.org/licenses/by/4.0/. The Creative Commons Public Domain Dedication waiver (http://creativeco mmons.org/publicdomain/zero/1.0/) applies to the data made available in this article, unless otherwise stated in a credit line to the data. 
competing for INCENP binding thereby promoting a spindle length necessary for successful asymmetric cell division [15]. In oocytes lacking AURKC, there is an INCENP-associated population of AURKA that compensates, and it is detectable by microscopy. In HeLa cells, AURKA co-immunoprecipitates with INCENP although it cannot be detected on chromosomes by immunocytochemistry [16]. These findings prompted us to consider whether there was a subpopulation of INCENP-bound AURKA in wild-type (WT) mouse oocytes that is undetectable by available methods of protein visualization.

To more accurately characterize AURK complexes, a tool that disrupts binding partner interactions rather than catalytic activity is necessary. The activator binding site inhibitor, ZINC08918027 (ZC), blocks the AURK::INCENP interaction by affinity for the INCENP binding site on the kinase in mitotic cells [17]. Here we examine the utility of $\mathrm{ZC}$ in disrupting $\mathrm{CPC}$ function in mouse oocytes.

\section{Main text \\ Materials and methods \\ Mice}

Female CF-1 mice (Envigo; Figs. 1, 3A, B) or CD-1 mice (Charles River Laboratories; Fig. 2 and Additional file 2: Video S1) between 6 and 8 weeks old were used for most experiments. Animals were euthanized via cervical dislocation without anesthesia to prevent stress hormone release. The generation and genotyping of Aurkb conditional knockout (cKO)/Aurkc knockout (KO) mice (Fig. 3C) were described previously [15]. Animals were housed in 12-12 h light-dark cycle, with constant temperature and with food and water provided ad libitum.

\section{Oocyte collection and maturation}

Female mice were injected intraperitoneally with 5 I.U. of pregnant mare's serum gonadotropin (Lee Biosolutions, \# 493-10). Prophase I-arrested oocytes were collected from both ovaries $48 \mathrm{~h}$ post-injection; oocyte numbers depended upon the experiment (indicated in legends) whereas live imaging experiments used at least 20 oocytes/treatment/replicate and western blotting used 100 oocytes/treatment per replicate. Oocytes from multiple mice were mixed together prior to splitting into experimental and control groups to reduce animalto-animal variation. 2.5 $\mu \mathrm{M}$ Milrinone (Sigma-Aldrich \#M4659) was added to bicarbonate free minimal essential medium (MEM) (25 mM Hepes, $\mathrm{pH} 7.3$, and $3 \mathrm{mg} /$ $\mathrm{mL}$ polyvinylpyrrolidone) to prevent the oocytes from resuming meiosis spontaneously during collection. Oocytes were cultured in Chatot, Ziomek, and Bavister medium without milrinone at $37{ }^{\circ} \mathrm{C}$ in $5 \% \mathrm{CO}_{2}$ [18]. For Metaphase I experiments, oocytes were cultured for
7.5 h. For Metaphase II experiments, oocytes were cultured for $16 \mathrm{~h}$. Organ culture dishes (Becton Dickinson \#353037) were used for in vitro maturation of drugtreated oocytes. At the end of the maturation period, the samples were fixed for immunostaining or frozen on dry ice for SDS-PAGE. All drug concentrations were prepared at a dilution factor of 1:2000.

For live-cell confocal imaging, prophase I-arrested oocytes were microinjected in M2 medium (SigmaAldrich) and cultured in MEM medium (Sigma-Aldrich) supplemented with $1.14 \mathrm{mM}$ sodium pyruvate (SigmaAldrich), $4 \mathrm{mg} / \mathrm{ml}$ bovine serum albumin (SigmaAldrich), $75 \mathrm{U} / \mathrm{ml}$ penicillin (Sigma-Aldrich) and $60 \mu \mathrm{g} /$ $\mathrm{ml}$ streptomycin (Sigma-Aldrich), at $37{ }^{\circ} \mathrm{C}$ in a $5 \% \mathrm{CO}_{2}$. Oocytes were stained with $100 \mathrm{nM}$ SiR-tubulin (Spirochrome) for microtubule visualization. After removing the cumulus cells, oocytes were microinjected in M2 medium with $10 \mathrm{pl}$ of $50 \mathrm{ng} / \mu \mathrm{l} \mathrm{H} 2 \mathrm{~b}$-mCherry, and $125 \mathrm{ng} / \mu \mathrm{l}$ Egfp-Cdk5rap2. Microinjected oocytes were cultured for $3 \mathrm{~h}$ in MEM medium supplemented with milrinone to allow protein expression prior to experimental procedures.

See video protocol [19] for detailed protocol methods for collection, injections and immunocytochemistry.

\section{Plasmids}

To generate cRNAs, plasmids were linearized and in vitro transcribed using a mMessage mMachine T3 (Ambion \#AM1348) and T7 kits (Ambion \#AM1344), according to manufacturer's protocol. The synthesized cRNAs were then purified using an RNAeasy kit (Qiagen \#74104) and stored at $-80{ }^{\circ} \mathrm{C}$. H2B-mCherry and $m E G F P-C d k 5 r a p 2$ cRNA constructs were described previously [20].

\section{Western blotting}

Oocytes were cleaned of cumulus cells with repetitive pipetting. Metaphase I oocytes (100/treatment) were mixed with sample buffer (1\% SDS, 1\% $\beta$-mercaptoethanol, 20\% glycerol, $50 \mathrm{mM}$ Tris- $\mathrm{HCl}$ (pH 6.8)) and phosphatase inhibitors sodium fluoride $(25 \mathrm{mM})$ and sodium orthovanadate $(1 \mathrm{mM})$ and denatured at $95{ }^{\circ} \mathrm{C}$ for $5 \mathrm{~min}$. Western blotting for pAURKA/ B/C (1:500; Cell Signaling Technologies \#2914) was conducted as detailed previously [7, 21]. The rabbit Tubulin antibody (Cell Signaling Technology, \#11H10) was used as a loading control.

\section{Immunocytochemistry}

Oocytes were fixed in phosphate-buffered saline containing 2\% paraformaldehyde (PFA; Sigma-Aldrich \#P6148) for $20 \mathrm{~min}$. Permeabilization and staining was conducted as described previously [19]. After final washes, the cells were mounted in VectaShield (Vector Laboratories, 


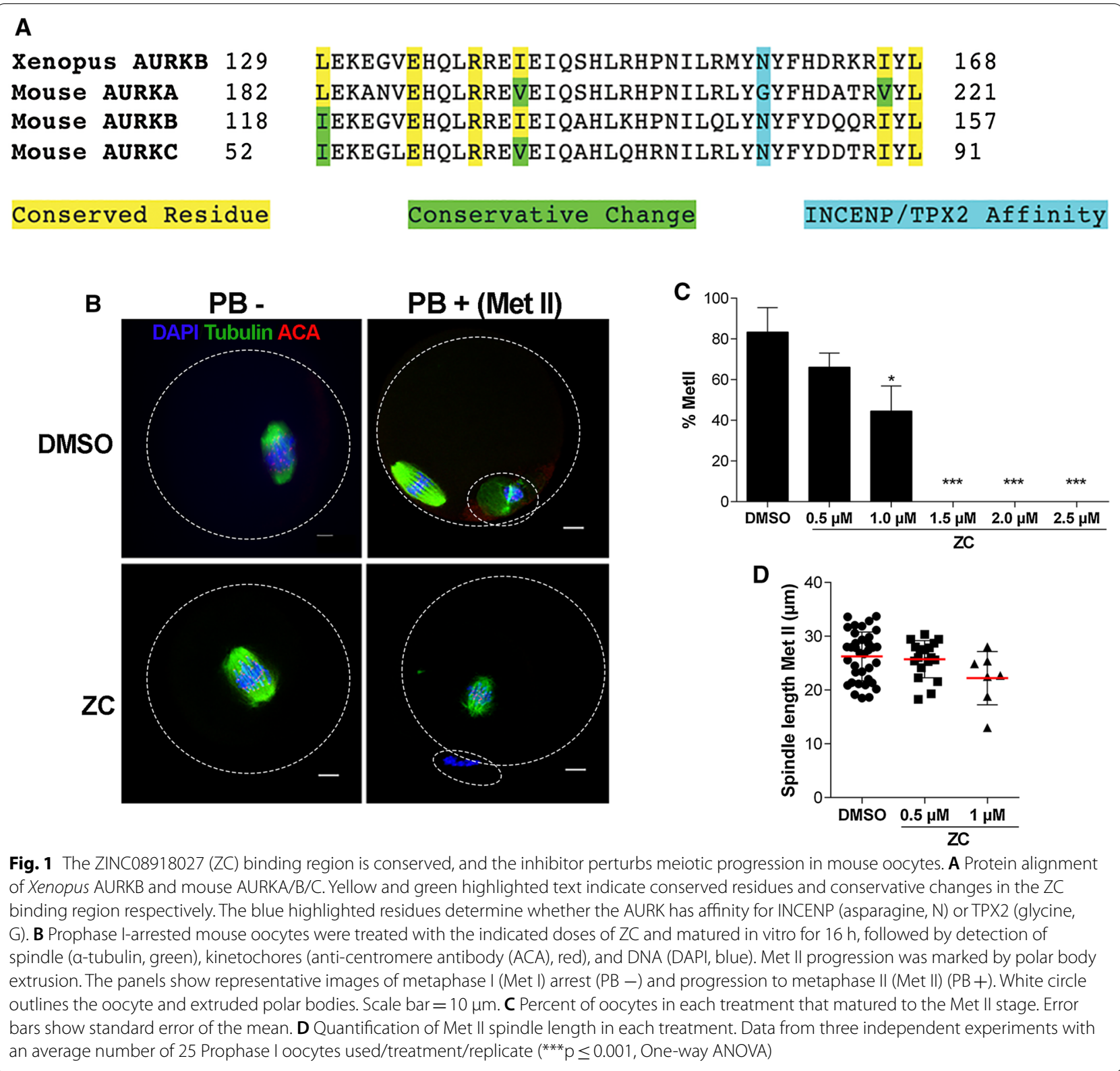

\#H-1000, Burlingame, USA) with 4', 6-Diamidino-2-Phenylindole, Dihydrochloride (DAPI; Life Technologies \#D1306; 1:170).

\section{Antibodies}

For immunofluorescence experiments, the primary antibodies were as follows: mouse anti $\alpha$-tubulin Alexafluor 488 conjugated (1:100, Life Technologies \#322588), rabbit anti-pINCENP (1:1000, gift from M. Lampson, University of Pennsylvania; [22]) and anti-centromeric antigen (ACA) (1:30, Antibodies Incorporated \#15234). The following secondary antibodies were used for immunofluorescence at a concentration of 1:200: antirabbit-Alexa568 (Life Technologies \#A11011) and goat anti-human-Alexa 568 (Life Technologies \#A21090).

\section{Microscopy}

A Leica TCS SP8 confocal microscope, using a $40 \times$ objective, was used to capture the images. Optical z-slices were obtained separately for each image using a $1.0 \mu \mathrm{m}$ step and a zoom setting of 3.5. The power of the laser was left unchanged for each cell if pixel intensities were to be compared. Samples were coded 


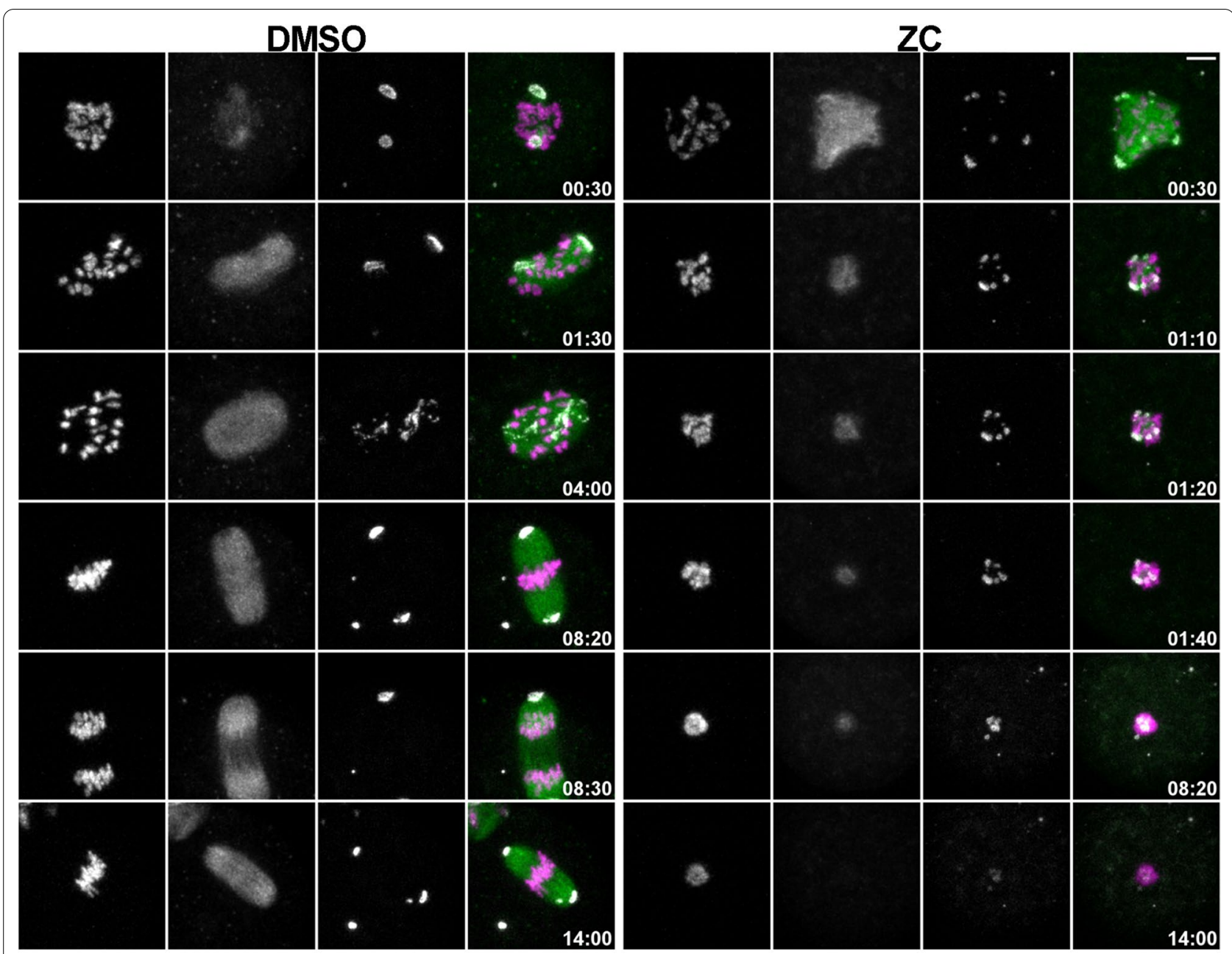

DNA MTOCs Microtubules

Fig. 2 Dynamics of spindle formation in ZINC08918027 (ZC)-treated oocytes. Still images from movies were taken at 10-min intervals. Time 0:30 represents the start of imaging from Prophase I arrest release. Time when each image was taken is noted in white beneath each image. DNA (H2B-mCHERRY; magenta), microtubule organizing centers (MTOCs, CDK5RAP2-EGFP; white), and microtubules (fluorogenic dye SiR-tubulin; green). Data from 3 replicates with 10 oocytes/treatment/replicate

so that images and analysis of treatment groups were obtained and conducted in a blinded fashion.

\section{Live cell imaging}

Time-lapse image acquisition was performed using a Leica TCS SP5 microscope with an HCX PL Apo Lambda Blue $40 \times 1.25$ NA oil objective. Oocytes were scanned using sequential scan in between line mode at a 12-bit image depth with $7.75 \times$ zoom on the chromosome area. 3D scanning was performed using $2.5-\mu \mathrm{m}$ optical sections through spindle volume. Image analysis was performed using FiJi software [23].

\section{Statistical analysis}

To evaluate differences between and among groups, a one-way analysis of variance (ANOVA) was used on Prism software (GraphPad). For all experiments, significance was marked by a p-value less than or equal to 0.05 . Experiments were performed in triplicate and oocyte number per replicate and/or total number is indicated in the figure legends.

\section{ZINC08918027 binding site sequence alignment}

The sequences of Xenopus laevis AURKB (accession number: AAM76715), Mus musculus AURKB (accession number: AAH03261), Mus musculus AURKC (accession 

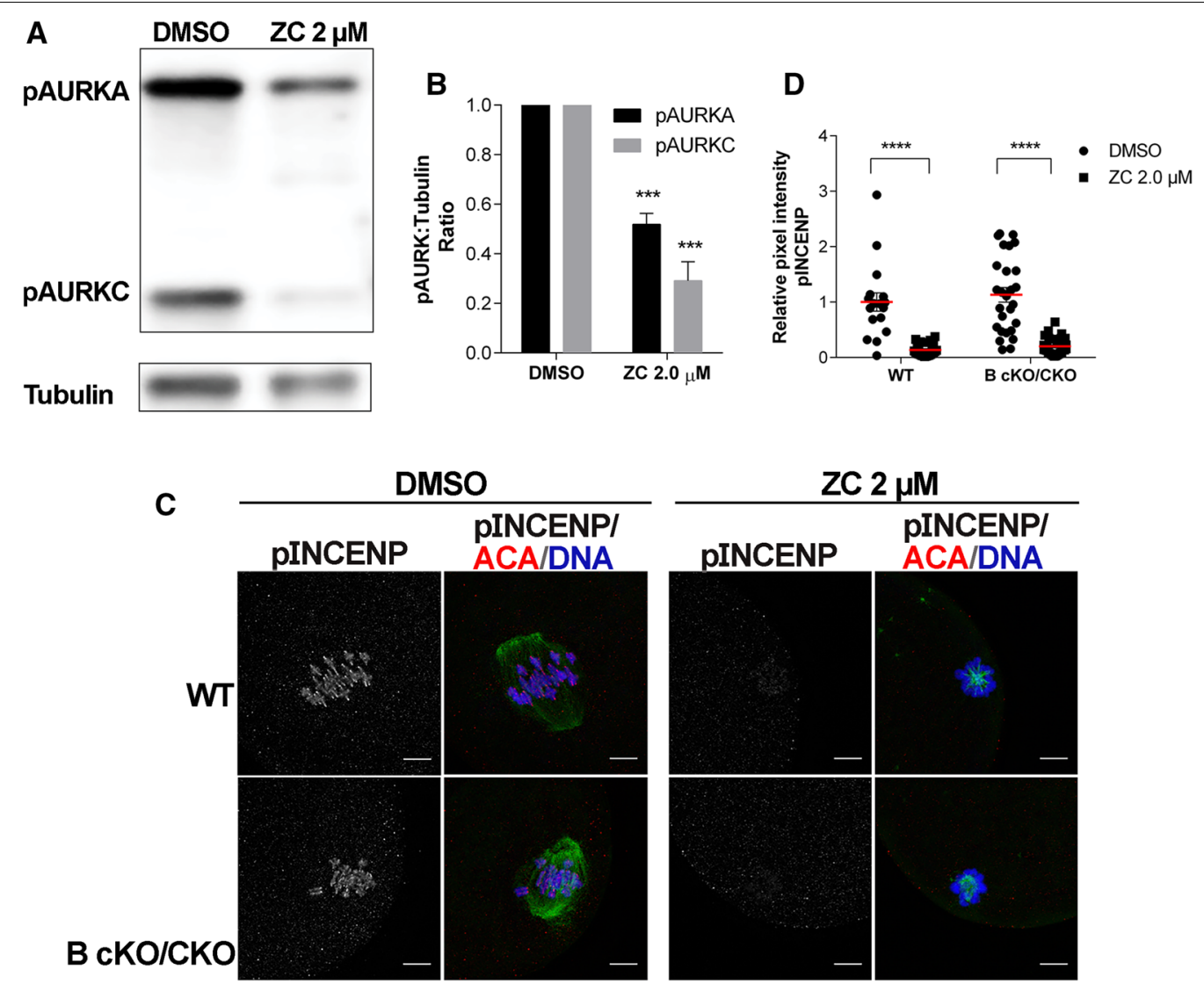

Fig. 3 ZINC08918027 (ZC) can inhibit both AURKA and AURKC. Prophase l-arrested mouse oocytes were treated with the indicated doses of ZC and matured in vitro to Metaphase I before freezing (A, B) or fixation (C). A Oocyte lysates (100 oocytes/per lane) were separated via SDS-PAGE and transferred to a membrane for western blotting to detect the activated forms of AURKA (pAURKA) and AURKC (pAURKC). Tubulin was used as a loading control. B Quantification of pAURKA and PAURKC normalized to Tubulin. Error bars show standard error of the mean. Data from three independent experiments ( ${ }^{* * *} p \leq 0.001$, (Unpaired Students t-Test, two-tailed)). C Wild-type (WT) or oocytes lacking both AURKB and AURKC were probed with anti-phosphorylated INCENP (pINCENP) antibodies (gray), a-tubulin (green) and mounted in DAPI (blue). Scale bar $=10 \mu \mathrm{m}$. D Quantification of integrated density of pINCENP from (C). WT DMSO $n=17$, WT Zn $2 \mu \mathrm{M}=25, \mathrm{~B} \mathrm{cKO} / \mathrm{C}$ KO DMSO n = 27, B cKO/C KO Zn $2 \mu M=33$

number: AAH64780), and Mus musculus AURKA (accession number: AAH14711) were aligned using the MacVector with Assembler software (MacVector, Inc.).

\section{Results and discussion}

Because all three AURK isoforms can bind INCENP [16, $24,25]$, we hypothesized that ZC could block the interaction between any AURK and INCENP in a cell type that expresses different AURK-containing CPC forms. $\mathrm{ZC}$ was designed to target the INCENP binding site on Xenopus laevis AURKB [17]. Therefore, to explore the similarities of this region between organisms, we aligned the ZC-binding region of Xenopus AURKB with the corresponding region in each mouse AURK. The alignment revealed that the $\mathrm{ZC}$-binding region was conserved in all three mouse isoforms (Fig. 1A). Residues in this binding region were either identical or were a biochemically conservative change. These analyses support our hypothesis that ZC can prevent binding between INCENP and any AURK isoform.

We then sought to determine the optimal concentration of $\mathrm{ZC}$ to use in mouse oocytes. The CPC is important for cytokinesis [26]. Therefore, we aimed to determine the lowest concentration of $\mathrm{ZC}$ at which polar body extrusion (PBE), the asymmetric cytokinesis in oocytes, was abolished indicating CPC disruption. We performed a dose-response curve and matured oocytes in vitro to Metaphase II (Met II) (Fig. 1B). The results showed that $\mathrm{PBE}$ decreased in a dose-dependent manner, with complete PBE failure beginning in $1.5 \mu \mathrm{M}$ $\mathrm{ZC}$ (Fig. 1C). In $1 \mu \mathrm{M} \mathrm{ZC,} \mathrm{PBE} \mathrm{was} \mathrm{reduced} \mathrm{by}>50 \%$ (Fig. 1C). The spindles of ZC-treated Met II eggs that did extrude polar bodies appeared shorter in length than the spindles of dimethylsulfoxide (DMSO)-treated oocytes (Fig. 1B, D). This finding is consistent with inhibition of AURKC-CPC and when AURKA is deleted [7, 9]. 
Because the spindle lengths of ZC-treated oocytes were shorter (Fig. 1D), we hypothesized that ZC adversely impacts spindle formation. To test this hypothesis, we conducted live imaging of oocyte maturation in the presence of $\mathrm{ZC}$ and chose a dose $(2.5 \mu \mathrm{M})$ where all oocytes failed to extrude a polar body (Fig. 1C). In control oocytes, the microtubule organizing centers (MTOCs) fragmented after nuclear envelope breakdown (NEBD), then clustered into two spindle poles before the chromosomes aligned at the metaphase plate, consistent with prior studies [27]. On the contrary, we observed that $100 \%$ of the oocytes treated with $\mathrm{ZC}$ never formed a bipolar spindle. We observed a disappearance of microtubule signal, and MTOCs gradually approached each other and moved towards the center of the oocyte. Furthermore, chromosomes did not align at the metaphase plate and instead condensed into a "ring-like" structure surrounding the cluster of MTOCs (Fig. 2; Additional file 2: Video S1). Therefore, we concluded that $\mathrm{ZC}$ disturbed spindle building in mouse oocytes. Taken together, these data demonstrate that $\mathrm{ZC}$ disrupts the formation of a bipolar spindle in mouse oocytes, which contributes to the reduction in PBE rates observed with $\mathrm{ZC}$ treatment.

$\mathrm{ZC}$ was designed with the intention to create an AURKB-specific inhibitor by blocking INCENP binding. However, because of the high degree of sequence conservation in this AURK domain (Fig. 1A) and because AURKA can bind INCENP in oocytes, we hypothesized that in oocytes ZC is not AURKC-specific and inhibits AURKA. To test this hypothesis, we first assessed AURKA/C activation. We treated WT oocytes with $2 \mu \mathrm{M}$ ZC and separated proteins from cell lysates by SDS-PAGE to resolve the activated forms of AURKA and AURKC from one another. In control-treated oocytes, activated AURKA (pAURKA) is more abundant than activated AURKC (pAURKC) (Fig. 3A, Additional file 1: Fig. S1). Upon ZC treatment, pAURKC declines 80\% (Fig. 3A, B), suggesting that both INCENP and non INCENP-bound populations of AURKC exist in oocytes. Importantly, we also saw a decline in PAURKA by $\sim 50 \%$ supporting the hypothesis that some AURKA is INCENP-bound in WT mouse oocytes. To further support this hypothesis, we used oocytes from mice lacking both Aurkb and Aurkc. We previously showed that in these double knockout oocytes, AURKA localizes to chromosomes, phosphorylates INCENP, and compensates for Aurkb/c loss [15]. These oocytes provide a simplified genetic background in which to visualize the impact of ZC on AURKA-CPC activity. To visualize AURKA-CPC activity, we probed oocytes with a phospho-specific INCENP antibody that detects the Aurora kinase specific phosphorylation sites [22]. WT and double knockout oocytes treated with
DMSO contained similar levels phosphorylated INCENP when we examined chromosomes at Met I (Fig. 3C, D). When WT oocytes were treated with $2 \mu \mathrm{M}$ ZC, pINCENP immunoreactivity was not detected. Similarly, pINCENP was absent when double Aurkb/c knockout oocytes with were treated with $2 \mu \mathrm{M}$ ZC (Fig. 3C, D). Taken together, these data indicate that $\mathrm{ZC}$ can prevent both AURKA and AURKC from binding INCENP and suggest that WT oocytes possess AURKA-CPC that is not visibly detectable, like some mitotic cell lines.

\section{Limitations}

Because oocytes are limiting in number, our studies are limited to assessing change in activity by immunofluorescence and western blotting. Therefore, we cannot isolate AURKA-INCENP and AURKA-TPX2 from oocytes to conduct biochemical analyses. Mouse oocytes also express AURKB, which is much less abundant and more challenging to detect. In our assays, we have not determined the impact ZC has on AURKB and this impact remains an open question. We also do not know if these results translate to human oocytes.

\section{Abbreviations \\ ACA: Anti-centromeric antigen; AURK: Aurora kinase; CKO: Conditional knock- out; CPC: Chromosomal passenger complex; DMSO: Dimethylsulfoxide; KO: Knockout; MEM: Minimal essential medium; MTOC: Microtubule organizing center; NEBD: Nuclear envelope breakdown; PBE: Polar body extrusion; ZC: ZINC08918027.}

\section{Supplementary Information}

The online version contains supplementary material available at https://doi. org/10.1186/s13104-022-05987-4.

Additional file 1: Figure S1. Full western blot image detecting the activated forms of AURKA (pAURKA) and AURKC (pAURKC) (top) and alphatubulin (bottom) from oocytes treated with ZC and matured to Met I. Red box: Area of cropping shown in Fig. 3A. Experiment repeated 3 times.

Additional file 2: Video S1. Dynamics of spindle formation in control and ZINC08918027 (ZC) treated oocytes. Time 0:00 represents the start of maturation from Prophase I arrest. DNA (H2B-mCHERRY), microtubule organizing centers (MTOCs, CDK5RAP2-EGFP), and microtubules (fluorogenic dye SiR-tubulin). $\mathrm{N}=10$ oocytes/treatment.

\section{Acknowledgements}

The authors thank members of the Schindler and Drutovic labs for helpful discussions. They acknowledge intellectual contribution from Dr. Petr Solc before his passing.

\section{Authors' contributions}

C.K. obtained funding, conducted experiments and data analysis, and wrote the manuscript. D.D. conducted experiments and edited the manuscript. C.S.B. mentored C.K. and edited the manuscript. K.S. conceived of and oversaw the project, obtained funding, and wrote and edited the manuscript. All authors read and approved the final manuscript.

\section{Funding}

This work was supported by an NIH grant R35 GM136340 to K.S., by an Aresty Foundation Research Grant to C.K and by the Inter-Excellence Program award 
(LTAUSA17097) to D.D. The granting agencies played no role in the design of the study and collection, analysis, and interpretation of data and in writing the manuscript.

\section{Availability of data and materials}

The imaging datasets used and/or analysed are available from the corresponding author upon request.

\section{Declarations}

\section{Ethics approval and consent to participate}

Animals were maintained following the Rutgers University Institutional Animal Use and Care Committee (Protocol 201702497), National Institutes of Health guidelines, and the policies of the Expert Committee for the Approval of Projects of Experiments on Animals of the Academy of Sciences of the Czech Republic (Protocol 43/2015). These regulatory bodies approved all experimental procedures involving animals prior to initiating the study.

\section{Consent for publication}

Not applicable.

\section{Competing interests}

The authors declare that they have no competing interests.

\section{Author details}

'Department of Genetics, Rutgers University, The State University of New Jersey, 145 Bevier Rd, Piscataway, NJ 08854, USA. ${ }^{2}$ Laboratory of DNA Integrity, Institute of Animal Physiology and Genetics of the Czech Academy of Sciences, Rumburska 89, Libechov, Czech Republic. ${ }^{3}$ Human Genetics Institute of NJ, Piscataway, NJ, USA.

Received: 12 November 2021 Accepted: 28 February 2022

Published online: 07 March 2022

\section{References}

1. Carmena M, Earnshaw WC. The cellular geography of aurora kinases. Nat Rev Mol Cell Biol. 2003;4(11):842-54.

2. Willems $E$, Dedobbeleer M, Digregorio M, Lombard A, Lumapat PN, Rogister B. The functional diversity of Aurora kinases: a comprehensive review. Cell Div. 2018;13:7.

3. Brown JR, Koretke KK, Birkeland ML, Sanseau P, Patrick DR. Evolutionary relationships of Aurora kinases: implications for model organism studies and the development of anti-cancer drugs. BMC Evol Biol. 2004;4(1):39.

4. Seeling JM, Farmer AA, Mansfield A, Cho H, Choudhary M. Differential selective pressures experienced by the Aurora kinase gene family. Int J Mol Sci. 2017. https://doi.org/10.3390/ijms19010072.

5. Yao LJ, Zhong ZS, Zhang LS, Chen DY, Schatten H, Sun QY. Aurora-A is a critical regulator of microtubule assembly and nuclear activity in mouse oocytes, fertilized eggs, and early embryos. Biol Reprod. 2004;70(5):1392-9.

6. Saskova A, Solc P, Baran V, Kubelka M, Schultz RM, Motlik J. Aurora kinase A controls meiosis I progression in mouse oocytes. Cell Cycle. 2008;7(15):2368-76

7. Blengini CS, Ibrahimian P, Vaskovicova M, Drutovic D, Solc P, Schindler K. Aurora kinase A is essential for meiosis in mouse oocytes. PLoS Genet. 2021;17(4): e1009327.

8. Yang KT, Li SK, Chang CC, Tang CJ, Lin YN, Lee SC, et al. Aurora-C kinase deficiency causes cytokinesis failure in meiosis I and production of large polyploid oocytes in mouse. Mol Biol Cell. 2010;21(14):2371-83.

9. Balboula $A Z$, Schindler K. Selective disruption of aurora $C$ kinase reveals distinct functions from aurora B kinase during meiosis in mouse oocytes. PLoS Genet. 2014;10(2): e1004194.

10. Kelly AE, Funabiki $\mathrm{H}$. Correcting aberrant kinetochore microtubule attachments: an Aurora B-centric view. Curr Opin Cell Biol. 2009;21(1):51-8.

11. Santaguida S, Vernieri C, Villa F, Ciliberto A, Musacchio A. Evidence that Aurora $B$ is implicated in spindle checkpoint signalling independently of error correction. EMBO J. 2011;30(8):1508-19.
12. Nezi L, Musacchio A. Sister chromatid tension and the spindle assembly checkpoint. Curr Opin Cell Biol. 2009;21 (6):785-95.

13. Blengini CS, Nguyen AL, Aboelenain M, Schindler K. Age-dependent integrity of the meiotic spindle assembly checkpoint in females requires Aurora kinase B. Aging Cell. 2021. https://doi.org/10.1111/acel.13489.

14. Aboelenain M, Schindler K. Aurora kinase B inhibits aurora kinase A to control maternal mRNA translation in mouse oocytes. Development. 2021. https://doi.org/10.1242/dev.199560.

15. Nguyen AL, Drutovic D, Vazquez BN, El Yakoubi W, Gentilello AS, Malumbres $\mathrm{M}$, et al. Genetic interactions between the aurora kinases reveal new requirements for AURKB and AURKC during oocyte meiosis. Curr Biol. 2018;28(21):3458-68 e5.

16. DeLuca KF, Meppelink A, Broad AJ, Mick JE, Peersen OB, Pektas S, et al. Aurora A kinase phosphorylates Hec1 to regulate metaphase kinetochore-microtubule dynamics. J Cell Biol. 2018;217(1):163-77.

17. Unsal E, Degirmenci B, Harmanda B, Erman B, Ozlu N. A small molecule identified through an in silico screen inhibits Aurora B-INCENP interaction. Chem Biol Drug Des. 2016;88(6):783-94.

18. Chatot CL, Ziomek CA, Bavister BD, Lewis JL, Torres I. An improved culture medium supports development of random-bred 1-cell mouse embryos in vitro. J Reprod Fertil. 1989;86(2):679-88.

19. Stein P, Schindler K. Mouse oocyte microinjection, maturation and ploidy assessment. JVis Exp. 2011. https://doi.org/10.3791/2851.

20. Balboula AZ, Nguyen AL, Gentilello AS, Quartuccio SM, Drutovic D, Solc P, et al. Haspin kinase regulates microtubule-organizing center clustering and stability through Aurora kinase C in mouse oocytes. J Cell Sci. 2016;129(19):3648-60.

21. Fellmeth JE, Gordon D, Robins CE, Scott RT Jr, Treff NR, Schindler K. Expression and characterization of three Aurora kinase $C$ splice variants found in human oocytes. Mol Hum Reprod. 2015;21(8):633-44.

22. Salimian KJ, Ballister ER, Smoak EM, Wood S, Panchenko T, Lampson MA, et al. Feedback control in sensing chromosome biorientation by the Aurora B kinase. Curr Biol. 2011;21(13):1158-65.

23. Schindelin J, Arganda-Carreras I, Frise E, Kaynig V, Longair M, Pietzsch T, et al. Fiji: an open-source platform for biological-image analysis. Nat Methods. 2012;9(7):676-82.

24. Abdul Azeez KR, Chatterjee S, Yu C, Golub TR, Sobott F, Elkins JM. Structural mechanism of synergistic activation of Aurora kinase $B / C$ by phosphorylated INCENP. Nat Commun. 2019;10(1):3166.

25. Katayama H, Sasai K, Kloc M, Brinkley BR, Sen S. Aurora kinase-A regulates kinetochore/chromatin associated microtubule assembly in human cells. Cell Cycle. 2008;7(17):2691-704.

26. Hadders MA, Lens SMA. Changing places: chromosomal passenger complex relocation in early anaphase. Trends Cell Biol. 2022;32(2):165-176.

27. Clift D, Schuh M. A three-step MTOC fragmentation mechanism facilitates bipolar spindle assembly in mouse oocytes. Nat Commun. 2015;6:7217.

\section{Publisher's Note}

Springer Nature remains neutral with regard to jurisdictional claims in published maps and institutional affiliations.

Ready to submit your research? Choose BMC and benefit from:

- fast, convenient online submission

- thorough peer review by experienced researchers in your field

- rapid publication on acceptance

- support for research data, including large and complex data types

- gold Open Access which fosters wider collaboration and increased citations

- maximum visibility for your research: over $100 \mathrm{M}$ website views per year

At BMC, research is always in progress.

Learn more biomedcentral.com/submissions 